\title{
Hematological parameters as early predictors of delayed neurological sequelae in acute carbon monoxide poisoning
}

\author{
Marwa M. Shahin ${ }^{1}$, Alzahraa A. Allam², Rasha A. Elkholy ${ }^{3}$, Heba I. Lashin ${ }^{1}$, \\ ${ }^{1}$ Forensic Medicine and Clinical Toxicology Department, Faculty of medicine, Tanta University, Tanta, Egypt. \\ ${ }^{2}$ Internal Medicine Department, Faculty of medicine, Tanta University, Tanta, Egypt. \\ ${ }^{3}$ Clinical Pathology Department, Faculty of Medicine, Tanta University, Tanta, Egypt.
}

\begin{abstract}
Introduction: Delayed neurological sequelae (DNS) are the most frequent morbidity after acute carbon monoxide (CO) poisoning. Therefore, their prevention and early prediction are the main goal of treatment. Aim of the work: This study aimed to investigate parameters based on peripheral complete blood cell count (CBC) as predictors of DNS in acute CO poisoning. Methodology: It included 67 acutely CO poisoned patients and 38 healthy volunteers. On admission, history, clinical examination and routine laboratory investigations were done. Ratios based on CBC counts were measured. Patients were followed up along 6 months for DNS signs. Results: The results revealed that total white blood cells (WBC) count, absolute neutrophil count \& percentage and median values of neutrophil-lymphocyte ratio (NLR) \& systemic immune inflammation index (SII) were significantly higher while lymphocytes percentage, absolute monocytic count and percentage were significantly lower in CO poisoned patients compared to control group. DNS-complicated patients had significantly lower total WBC count, absolute neutrophil count \& percentage and significantly higher median values of NLR and SII than non-complicated. Based on receiver operating characteristic analysis (ROC), NLR and SII were significant predictors of DNS. Conclusion: It was concluded that, NLR and SII ratios may be helpful predictors of DNS after acute CO poisoning.
\end{abstract}

Key words Carbon monoxide poisoning, neutrophils, systemic immune inflammation index, delayed neurological sequelae, prognosis

\section{Introduction}

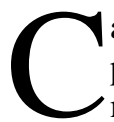
arbon monoxide (CO) is a known highly toxic gas produced by incomplete combustion of organic materials, with specific properties as it is colorless, odorless, tasteless, non-irritant gas (Wang et al., 2019). Acute CO poisoning remains an important cause of morbidity and mortality and represents a major public health problem (Liao et al., 2019b). About 50,000 cases with 1200 deaths for non-fire CO exposures were reported annually in the United States (Kim et al., 2019).

The affinity of CO for hemoglobin is 250 times much higher than that of oxygen (Eichhorn et al., 2018). Therefore, CO binds to hemoglobin rapidly after exposure to form carboxy-hemoglobin $(\mathrm{COHb})$ that has lower oxygen-carrying capacity leading to tissue hypoxia, oxidative stress and inflammation (Huysal et al., 2016). Additionally, CO causes free oxygen radicals formation and lipid peroxidation in different body tissues causing changes in the morphology and function of those organs (Cevik et al., 2010).

Brain and nervous system are the most injured organs by tissue hypoxia induced by acute $\mathrm{CO}$ poisoning causing delayed neurological sequelae (DNS) (Pang et al., 2013). It includes many symptoms and signs (recurrent headache, delirium, amnesia, cognitive dysfunction, personality changes, psychosis, urine and fecal incontinence) (Sönmez et al., 2018). After an initial clinical recovery from acute CO poisoning, DNS had been detected within 2 to 6 weeks in 3\% to $40 \%$ of cases. It may resolve gradually within the first months but unfortunately it can be permanent in $25 \%$ of cases. So, early prediction and prevention of DNS after acute CO poisoning are considered nowadays the main line of treatment (Guan et al., 2015; Jeon et al., 2018; Lin et al., 2018).

Although exact pathophysiology of DNS remains unclear, systemic inflammation process was found to have important role in the occurrence of DNS and fatal complications due to acute CO poisoning (Moon et al., 2019a). Following CO exposure, significant increase in leukocyte sequestration in brain microvasculature was detected. Additionally, the flux of nitric oxide released from platelets increases due to $\mathrm{CO}$ poisoning that may stimulate heterotypic (platelet-neutrophil) aggregation (Thom et al., 2006b; Karabacak et al., 2015). Furthermore, leukocytosis, neutrophilia, monocytosis, and lymphocytopenia have been detected in the acute phase of clinical conditions in which oxidative stress is increased (Kim et al., 2015). 
Moreover, no correlation has been found between $\mathrm{COHb}$ level in the blood and clinical symptoms or longterm prognosis of acute $\mathrm{CO}$ poisoning. This suggests that other processes rather than hypoxia such as inflammation may be indicative of occurrence of DNS and later complications. In particular, laboratory markers of inflammation would be helpful for clinicians to predict DNS after acute CO poisoning (Vezzani et al., 2016).

Recently, neutrophil-lymphocyte ratio (NLR), monocyte-lymphocyte ratio (MLR) and plateletlymphocyte ratio (PLR) are new simple inflammatory biomarkers which can be obtained easily based on peripheral complete blood cell (CBC) counts. They are useful prognostic marker in various clinical conditions with inflammatory responses (Kim et al., 2015; Moon et al., 2019b). Systemic immune inflammation index (SII) is a new inflammatory marker which was found to be a more objective prognostic marker as it reflects the balance between inflammatory and immune response of the body (Hu et al., 2014; Geng et al., 2016).

Hyperbaric oxygen (HBO) therapy is the primary method of treatment for acute severe CO poisoning due to its beneficial effect in preventing inflammatory changes in the brain and lipid peroxidation, consequently decreasing DNS rates (Vezzani et al., 2016; Hafez and El-Sarnagawy, 2019).

Although, association between systemic inflammation and DNS in acute CO poisoning was proved (Moon et al., 2019a) and there are multiple ratios based on peripheral CBC count available as new inflammatory prognostic markers, there are limited studies exploring the predictive value of these ratios for DNS in acute CO poisoning.

So, this study aimed to investigate whether hematological parameters based on peripheral CBC counts are associated with occurrence of DNS after acute CO poisoning and which parameters could predict them.

\section{Patients and Methods}

\section{Study design}

This prospective cohort study was carried out on all acute CO intoxicated patients who were admitted to Tanta University Poison Control Center (TUPCC) in the period from the start of November 2017 to the end of October 2018. Age and sex matched non-exposed 38 healthy volunteers (control group) were also included.

This study was performed after approval by the Research Ethics Committee (REC) of Faculty of Medicine, Tanta University (approval number: 31900/11/17). A written informed consent was explained and signed from each patient or from guardian of unconscious patients prior to the start of the study. Participants were anonymized with strict consideration of the confidentiality of personal and clinical data.

\section{Eligibility criteria}

Inclusion criteria: All patients of both sexes above 18 years with acute $\mathrm{CO}$ poisoning were included in this study. Diagnosis of acute CO intoxication was established based on history of CO exposure, clinical findings of poisoning (such as headache, nausea, vomiting, dizziness, malaise, alteration in consciousness level, syncope, seizures, shortness of breath, chest pain and palpitation). Elevated $\mathrm{COHb}$ level $>3-4 \%$ in nonsmokers or $>10 \%$ in smokers confirmed the diagnosis (Hampson et al., 2012; Rose et al., 2017).

Exclusion criteria: Patients with history of coingestion of other poisons, patients with risk factors such as pregnancy, chronic chest diseases e.g. asthma and chronic hematological diseases e.g. anemia as well as patients transferred from other hospitals were excluded. Moreover, patients with previous history of a neuropsychiatric disease or impaired cerebral perfusion and patients who had any disease that may affect white blood cells (WBC) count such as obesity, diabetes mellitus, malignancy, acute or chronic renal and liver disease, acute or chronic inflammatory disease, autoimmune disease, atherosclerotic heart diseases, valvular heart disease, heart failure, peripheral arterial disease were also excluded.

\section{Data collection and definition of variables}

Demographics (age and sex), medical and toxicological history (delay period between exposure and hospital arrival and duration of exposure) were recorded for each patient in data collection sheet. Clinical examination including Glasgow coma score (GCS), vital signs, neurological, respiratory and cardiovascular examination was done at admission for each patient.

All patients were treated by administering $100 \%$ normobaric oxygen by a non-rebreather mask upon arrival at hospital. The decision to refer patients to HBO therapy unit at Tanta University Educational Hospital was made by the treating physicians at TUPCC following the guidelines of $\mathrm{HBO}$ therapy for $\mathrm{CO}$ poisoning that were loss of consciousness on admission, neurological deficits, ischemic cardiac changes, significant metabolic acidosis and elevated $\mathrm{COHb}$ level $\geq 25 \%$ (Liao et al., 2019a). Number of needed HBO sessions and duration of hospital stay were registered.

\section{Blood sample and biochemical investigations}

Blood samples were collected immediately after admission under complete aseptic conditions and before giving any medication for arterial blood gases and peripheral CBC counts analysis. Peripheral CBC ratios were calculated as follows: SII $=$ platelet count $\times$ neutrophil count/lymphocyte count (Hu et al., 2014); NLR = neutrophil count/lymphocyte count; MLR = monocyte count/lymphocyte count; and PLR = platelet count/lymphocyte count.

The Rad-57 Pulse CO-Oximeter (Masimo SET Rainbow, Irvine, California, USA) depending on a multiwave length sensor was used as a continuous and noninvasive method of measuring $\mathrm{COHb}$ level in blood at admission. Twelve-lead electrocardiography (ECG) was recorded for each patient at admission and corrected QT (QTc) interval was detected according to the Bazett's 
formula; QTc $=\mathrm{QT} / \sqrt{\mathrm{RR}}$ (Normally, QTc is $\leq 440 \mathrm{msec}$ ) (Postema and Wilde, 2014).

ECG changes induced by acute CO poisoning were graded according to poisoning severity score (Akdur et al., 2010) into: Minor ECG changes: Isolated extrasystoles, sinus tachycardia (HR $\geq 100-140$ in adults). Moderate ECG changes: Sinus tachycardia (HR $=140-180$ in adults), frequent extrasystoles, atrial fibrillation/flutter, AV-block I-II, prolonged QRS and QTc-time, repolarization abnormalities or myocardial ischaemia. Severe ECG changes: severe sinus tachycardia ( $\mathrm{HR} \geq 180$ in adults), life-threatening ventricular dysrythmias, AV block III, asystole, myocardial infarction.

\section{Follow up for DNS}

Assessment of acute $\mathrm{CO}$ poisoned patient's neurological and psychological outcome was done initially after patient regain stable level of consciousness. Then, all patients were invited for follow up regular visits after being discharged for detection of any manifestations that referred as DNS and patients with any detected DNS were directed to have neuropsychiatric consultation. The recommended follow up duration was at least 6 months after acute CO poisoning. Symptoms of DNS were recorded in a simple questionnaire including leading questions.

Occurrence of DNS in the included patients was confirmed by recurrence of original neurological or psychological symptoms or by development of new ones. The symptoms included one or more of the following: difficulty concentrating, lethargy, emotional liability, amnestic syndromes, dementia, cognitive impairment, personality changes, learning difficulties, psychosis, depression, Parkinsonism, apraxia, gait disturbance, urinary incontinence (Pepe et al., 2011; Cha et al., 2018).

\section{Statistical Analysis}

Acute $\mathrm{CO}$ poisoned patients were divided into two groups according to appearance of DNS after co poisoning: DNS-complicated and non-complicated patients. Collected data were organized and entered to the computer using SPSS (Statistical Package for Social Science) program for statistical analysis (version 22). Data were entered as numerical or categorical, as appropriate. Kolmogorov-Smirnov test of normality revealed significance in the distribution of some variables, so the non-parametric statistics were adopted. Minimum, maximum, median and inter-quartile range (IQR) were calculated. Chi-Squared test (Monte-Carlo corrected) for NxM table. Receiver operating characteristic (ROC) analysis was generated from the data for a discriminative cut-off value for predicting the occurrence of DNS. Sensitivity and specificity were also calculated using Medcalc software. Area under ROC curve (AUC) was graded as follows: $0.90-1=$ excellent; 0.80-0.90 = good; $0.70-0.80=$ fair; and 0.60-0.70 = poor.

\section{Results}

Demographics, vital signs and QTc interval of acute $\mathrm{CO}$ poisoned patients were compared to control group as shown in table (1). Age and sex were comparable in both groups with no statistical difference. Blood pressure (both systolic and diastolic) and oxygen saturation were significantly lower while QTc interval was significantly longer in $\mathrm{CO}$ poisoned patients.

Concerning hematological parameters (table 2), data obtained by mann-whitney test revealed that haemoglobin level, total WBC count, absolute neutrophil count and percentage were significantly higher while lymphocytes percentage, absolute monocytic count and percentage were significantly lower in $\mathrm{CO}$ poisoned patients than control group. Moreover, median values of NLR and SII were significantly higher in $\mathrm{CO}$ poisoned patients (3.35 and 869.6 respectively) when compared with control group (1.92 and 488.8 respectively) while; MLR and PLR did not show any statistical difference between them.

DNS had developed in 33 patients $(53.2 \%)$. The frequency of detected symptoms within DNS-complicated patients were recurrent headache and memory loss $(27.3 \%$ each), difficult speech and concentration deficit $(24.2 \%$ each), personality changes (18.2\%), insomnia and gait disturbance $(9.1 \%$ each).

Comparisons of demographics, admission data, vital signs, QTc, ECG severity grading, $\mathrm{COHb}$ level, hospital stay and number of needed HBO sessions between DNS-complicated and non-complicated patients were done (Table 3,4). DNS-complicated patients had significantly lower initial GCS and oxygen saturation and higher $\mathrm{COHb}$ level on admission, length of hospital stay and number of needed HBO sessions than noncomplicated ones.

Table (5) showed that DNS-complicated patients had significant higher median values of total WBC count, absolute neutrophil count and percentage and lower median value of lymphocytes percentage than noncomplicated ones. NLR and SII median values were significantly higher in DNS-complicated patients (4.85 and 1112.4 respectively) than non-complicated ones (2.51 and 730.3 respectively) while; MLR and PLR did not show any statistical difference between them.

Based on ROC analysis (figure 2,3), both of NLR and SII were significant predictors of DNS in acute CO poisoned patients. For NLR, $\mathrm{AUC}=0.711((95 \%$ CI 0.581-0.819) $\quad(\mathrm{Z}=3.141, \quad \mathrm{p}=0.0017)$. The diagnostic criterion using Youden index is the level of $>2.69$ with a sensitivity of $78.8 \%$ (95\% CI $61.1-91)$, specificity of $62.1 \%(95 \%$ CI $42.3-79.3)$, positive predictive value (PPV) of $70.3 \%$ and negative predictive value (NPV) of $72 \%$. While for SII, AUC $=0.741(95 \%$ CI $0.614-0.844)$ $(\mathrm{Z}=3.866, \mathrm{p}=0.0001)$. The diagnostic criterion using Youden index is the level of $>934.93\left(\times 10^{3} / \mathrm{mm}^{3}\right)$ with a sensitivity of $63.6 \%$ (95\% CI 45.1-79.6), specificity of $75.9 \%$ (95\% CI $56.5-89.7)$, PPV of $75 \%$ and NPV of $64.7 \%$. 
Table (1): Demographics, vital signs, arterial blood gases and QTc interval of acute CO poisoned patients at admission and control group.

\begin{tabular}{|c|c|c|c|c|c|}
\hline \multicolumn{2}{|c|}{ Variables } & $\begin{array}{c}\text { Acute } \mathrm{CO} \\
\text { poisoned patients } \\
(\mathrm{n}=62)\end{array}$ & $\begin{array}{c}\text { Control group } \\
(\mathbf{n}=\mathbf{3 8 )}\end{array}$ & $\begin{array}{c}\text { Test of } \\
\text { significance }\end{array}$ & $p$ value \\
\hline Age (years) & $\begin{array}{c}\text { Min-Max } \\
\text { Median (IQR) }\end{array}$ & $\begin{array}{c}18-75 \\
24(20-35)\end{array}$ & $\begin{array}{c}18-70 \\
25(21-32)\end{array}$ & $\mathrm{Z}_{(\mathrm{MW})}=-0.210$ & 0.834 \\
\hline $\begin{array}{ll}\text { Sex: } & \text { Males } \\
& \text { Females }\end{array}$ & $\begin{array}{l}\mathrm{N}(\%) \\
\mathrm{N}(\%)\end{array}$ & $\begin{array}{l}34(54.8 \%) \\
28(45.2 \%)\end{array}$ & $\begin{array}{l}20(52.6 \%) \\
18(47.4 \%)\end{array}$ & $\mathrm{X}_{(\mathrm{df}=1)}^{2}=0.046$ & 0.830 \\
\hline $\begin{array}{l}\text { Systolic blood pressure } \\
\text { (mmHg) }\end{array}$ & $\begin{array}{c}\text { Min-Max } \\
\text { Median (IQR) }\end{array}$ & $\begin{array}{c}80-150 \\
110(100-120)\end{array}$ & $\begin{array}{c}90-150 \\
120(110-130)\end{array}$ & $\mathrm{Z}_{(\mathrm{MW})}=-3.189$ & $0.001 *$ \\
\hline $\begin{array}{l}\text { Diastolic blood pressure } \\
\text { (mmHg) }\end{array}$ & $\begin{array}{c}\text { Min-Max } \\
\text { Median (IQR) }\end{array}$ & $\begin{array}{c}50-100 \\
65(60-70) \\
\end{array}$ & $\begin{array}{c}60-100 \\
75(70-80) \\
\end{array}$ & $\mathrm{Z}_{(\mathrm{MW})}=-2.988$ & $0.003 *$ \\
\hline Pulse (beats/min) & $\begin{array}{c}\text { Min-Max } \\
\text { Median (IQR) }\end{array}$ & $\begin{array}{c}67-153 \\
103(93-120)\end{array}$ & $\begin{array}{c}75-132 \\
100(89-114)\end{array}$ & $\mathrm{Z}_{(\mathrm{MW})}=-1.174$ & 0.240 \\
\hline $\begin{array}{l}\text { Respiratory rate } \\
\text { (breaths/min) }\end{array}$ & $\begin{array}{c}\text { Min-Max } \\
\text { Median (IQR) }\end{array}$ & $\begin{array}{c}12-40 \\
18.5(16-23)\end{array}$ & $\begin{array}{c}14-26 \\
20(17-22)\end{array}$ & $\mathrm{Z}_{(\mathrm{MW})}=-0.545$ & 0.586 \\
\hline Temperature $\left({ }^{\circ} \mathrm{C}\right)$ & $\begin{array}{c}\text { Min-Max } \\
\text { Median (IQR) }\end{array}$ & $\begin{array}{c}36-38 \\
37.2(37-37.2)\end{array}$ & $\begin{array}{c}36.5-37.5 \\
37.05(37-37.2)\end{array}$ & $\mathrm{Z}_{(\mathrm{MW})}=-1.243$ & 0.214 \\
\hline Oxygen saturation (\%) & $\begin{array}{c}\text { Min-Max } \\
\text { Median (IQR) }\end{array}$ & $\begin{array}{c}40-100 \\
97(89-99)\end{array}$ & $\begin{array}{c}95-100 \\
98.5(98-99)\end{array}$ & $\mathrm{Z}_{(\mathrm{MW})}=-3.341$ & $0.001 *$ \\
\hline pH & $\begin{array}{c}\text { Min-Max } \\
\text { Median (IQR) }\end{array}$ & $\begin{array}{c}7.22-7.57 \\
7.42(7.37-7.46)\end{array}$ & $\begin{array}{c}7.37-7.55 \\
7.43(7.40-7.46)\end{array}$ & $\mathrm{Z}_{(\mathrm{MW})}=-1.435$ & 0.151 \\
\hline $\mathrm{PCO}_{2}(\mathrm{mmHg})$ & $\begin{array}{c}\text { Min-Max } \\
\text { Median (IQR) }\end{array}$ & $\begin{array}{c}22-330 \\
35.55(31.7-38.9)\end{array}$ & $\begin{array}{c}25.1-48 \\
34.9(32.1-37.5)\end{array}$ & $\mathrm{Z}_{(\mathrm{MW})}=-0.472$ & 0.637 \\
\hline QTc (msec) & $\begin{array}{c}\text { Min-Max } \\
\text { Median (IQR) }\end{array}$ & $\begin{array}{c}360-516 \\
422.5(404-465) \\
\end{array}$ & $\begin{array}{c}341-480 \\
408(390-424)\end{array}$ & $Z_{(M W)=}-2.881$ & $0.004 *$ \\
\hline $\begin{array}{l}\text { QTc interval (Category): } \\
\text { Normal } \\
\text { Prolonged (>440msec) }\end{array}$ & $\begin{array}{l}\mathrm{N}(\%) \\
\mathrm{N}(\%)\end{array}$ & $\begin{array}{l}36(58.1 \%) \\
26(41.9 \%)\end{array}$ & $\begin{array}{c}33(86.8 \%) \\
5(13.2 \%)\end{array}$ & $X^{2}(d f=1)=9.122$ & $0.003 *$ \\
\hline
\end{tabular}

Min-Max: Minimum-Maximum; IQR: inter-quartile range ( $25^{\text {th }}-75^{\text {th }}$ percentile); MW: Mann-Whitney test

*: Statistically significant $(p<0.05)$; \%: Percent within group; $d f:$ : degree of freedom

Table (2): Hematologic parameters based on peripheral CBC count of acute CO poisoned patients at admission and control group.

\begin{tabular}{|c|c|c|c|c|c|}
\hline \multicolumn{2}{|c|}{ Variables } & $\begin{array}{l}\text { Acute CO poisoned } \\
\text { patients }(n=62)\end{array}$ & $\begin{array}{c}\text { Control group } \\
(n=38)\end{array}$ & $\mathbf{Z}_{(\mathrm{MW})}$ & $p$ value \\
\hline Hb (\%) & $\begin{array}{c}\text { Min-Max } \\
\text { Median (IQR) }\end{array}$ & $\begin{array}{c}9.1-15.6 \\
12.8(12-13.8)\end{array}$ & $\begin{array}{c}8.2-15.4 \\
11.9(10.7-12.7)\end{array}$ & -3.251 & $0.001^{*}$ \\
\hline Platelets $\left(\mathrm{x}^{10} / \mathrm{mm}^{3}\right)$ & $\begin{array}{c}\text { Min-Max } \\
\text { Median (IQR) }\end{array}$ & $\begin{array}{c}90-380 \\
242(201-298)\end{array}$ & $\begin{array}{c}124-398 \\
229(195-278)\end{array}$ & -0.856 & 0.392 \\
\hline $\begin{array}{l}\text { White blood cells } \\
\text { (cells/mm³) }\end{array}$ & $\begin{array}{c}\text { Min-Max } \\
\text { Median (IQR) }\end{array}$ & $\begin{array}{c}48000-19800 \\
8400(6500-11500)\end{array}$ & $\begin{array}{c}3700-11000 \\
6350(5200-7900)\end{array}$ & -3.723 & $0.000 *$ \\
\hline Neutrophil (cells/mm³) & $\begin{array}{c}\text { Min-Max } \\
\text { Median (IQR) }\end{array}$ & $\begin{array}{c}2706-17150 \\
6130(4466-9500)\end{array}$ & $\begin{array}{c}1730.4-7242 \\
3938(3299-5325)\end{array}$ & -4.566 & $0.000^{*}$ \\
\hline Neutrophil (\%) & $\begin{array}{c}\text { Min-Max } \\
\text { Median (IQR) }\end{array}$ & $\begin{array}{c}52-89 \\
74.5(64-82)\end{array}$ & $\begin{array}{c}40-77 \\
61.05(56-70)\end{array}$ & -4.968 & $0.000 *$ \\
\hline $\begin{array}{l}\text { Absolute lymphocytic } \\
\text { count (cells/mm } / \mathbf{m m}^{3} \text { ) }\end{array}$ & $\begin{array}{c}\text { Min-Max } \\
\text { Median (IQR) }\end{array}$ & $\begin{array}{c}952-3468 \\
1878(1748-2130)\end{array}$ & $\begin{array}{c}1097.6-6050 \\
1929(1456-2556)\end{array}$ & -0.575 & 0.565 \\
\hline Lymphocyte (\%) & $\begin{array}{c}\text { Min-Max } \\
\text { Median (IQR) }\end{array}$ & $\begin{array}{c}10-44 \\
23(15-32)\end{array}$ & $\begin{array}{c}17-55 \\
31.9(25-38)\end{array}$ & -4.154 & $0.000^{*}$ \\
\hline $\begin{array}{l}\text { Absolute monocytic } \\
\text { count (cells/mm } \text { mm }^{3} \text { ) }\end{array}$ & $\begin{array}{c}\text { Min-Max } \\
\text { Median (IQR) }\end{array}$ & $\begin{array}{c}48-1068 \\
225.5(126-380)\end{array}$ & $\begin{array}{c}80-756 \\
298(240.1-425)\end{array}$ & -2.351 & $0.019 *$ \\
\hline Monocyte (\%) & $\begin{array}{c}\text { Min-Max } \\
\text { Median (IQR) } \\
\end{array}$ & $\begin{array}{c}1-9 \\
2.5(1-5) \\
\end{array}$ & $\begin{array}{c}2-9 \\
5(4-5) \\
\end{array}$ & -4.085 & $0.000 *$ \\
\hline
\end{tabular}


Table (2): Continued

\begin{tabular}{|c|c|c|c|c|c|}
\hline \multicolumn{2}{|c|}{ Variables } & $\begin{array}{l}\text { Acute CO poisoned } \\
\text { patients }(n=62)\end{array}$ & $\begin{array}{c}\text { Control group } \\
(n=38)\end{array}$ & $\mathrm{Z}_{(\mathrm{MW})}$ & $p$ value \\
\hline NLR & $\begin{array}{c}\text { Min-Max } \\
\text { Median (IQR) }\end{array}$ & $\begin{array}{c}1.18-8.91 \\
3.35(1.94-5.3)\end{array}$ & $\begin{array}{c}0.73-4.53 \\
1.92(1.55-2.84)\end{array}$ & -4.417 & $0.000 *$ \\
\hline MLR & $\begin{array}{c}\text { Min-Max } \\
\text { Median (IQR) } \\
\end{array}$ & $\begin{array}{c}0.03-0.69 \\
0.12(0.06-0.22) \\
\end{array}$ & $\begin{array}{c}0.04-0.32 \\
0.15(0.11-0.22) \\
\end{array}$ & -1.631 & 0.103 \\
\hline PLR & $\begin{array}{c}\text { Min-Max } \\
\text { Median (IQR) }\end{array}$ & $\begin{array}{c}38.06-363.98 \\
134(108.06-160.56)\end{array}$ & $\begin{array}{c}37.96-307.94 \\
117.64(96.26-146.48) \\
\end{array}$ & -1.520 & 0.129 \\
\hline $\mathrm{SII}\left(\mathrm{x} 10^{3} / \mathrm{mm}^{3}\right)$ & $\begin{array}{c}\text { Min-Max } \\
\text { Median (IQR) }\end{array}$ & $\begin{array}{c}168.75-3041.46 \\
869.64(509.13-1327.80)\end{array}$ & $\begin{array}{c}166.76-1096.99 \\
488.82(330-619.11)\end{array}$ & -4.282 & $0.000 *$ \\
\hline
\end{tabular}

Min-Max: Minimum-Maximum; *: Statistically significant ( $p<0.05)$; IQR: inter-quartile range $\left(25^{\text {th }}-75^{\text {th }}\right.$ percentile) MW: Mann-Whitney test

Table (3): Demographics, admission data, QTc, ECG severity grading, hospital stay and number of needed HBO sessions of DNS-complicated and non-complicated acute CO poisoned patients.

\begin{tabular}{|c|c|c|c|c|c|}
\hline \multirow{2}{*}{\multicolumn{2}{|c|}{ Variables }} & \multicolumn{2}{|c|}{ Acute $\mathrm{CO}$ poisoned patients } & \multirow{3}{*}{$\begin{array}{c}\begin{array}{c}\text { Test of } \\
\text { significance }\end{array} \\
\mathrm{Z}_{(\mathrm{MW})=-0.955}\end{array}$} & \multirow{3}{*}{$\begin{array}{r}\boldsymbol{p} \text { value } \\
0.340\end{array}$} \\
\hline & & \multirow{2}{*}{$\begin{array}{c}\begin{array}{c}\text { Non-complicated } \\
\text { patients } \\
\text { (n=29, } \\
\mathbf{4 6 . 8 \%})\end{array} \\
18-75 \\
25(21-35)\end{array}$} & \multirow{2}{*}{$\begin{array}{c}\begin{array}{c}\text { DNS-complicated } \\
\text { patients } \\
\text { (n=33, 53.2\%) }\end{array} \\
18-48 \\
23(20-35)\end{array}$} & & \\
\hline Age (years) & $\begin{array}{c}\text { Min-Max } \\
\text { Median (IQR) }\end{array}$ & & & & \\
\hline $\begin{array}{l}\text { Sex: Males } \\
\text { Females }\end{array}$ & $\begin{array}{l}\text { N (\%) } \\
\text { N (\%) }\end{array}$ & $\begin{array}{l}11(37.93 \%) \\
18(62.07 \%)\end{array}$ & $\begin{array}{l}23(69.7 \%) \\
10(30.3 \%)\end{array}$ & $X_{(d f=1)}^{2}=6.289$ & $0.012 *$ \\
\hline Delay (hours) & $\begin{array}{c}\text { Min-Max } \\
\text { Median (IQR) }\end{array}$ & $\begin{array}{l}0.5-36 \\
3(1-4)\end{array}$ & $\begin{array}{l}0.5-36 \\
2(1-5)\end{array}$ & $Z_{(\mathrm{MW})=-}-0.546$ & 0.585 \\
\hline $\begin{array}{c}\text { Duration of exposure } \\
\text { (hours) }\end{array}$ & $\begin{array}{c}\text { Min-Max } \\
\text { Median (IQR) }\end{array}$ & $\begin{array}{l}0.5-12 \\
2(1-4)\end{array}$ & $\begin{array}{c}0.5-15 \\
4.5(2-7)\end{array}$ & $\mathrm{Z}_{(\mathrm{MW})=-2.662}$ & 0.008 \\
\hline Initial GCS on admission & $\begin{array}{c}\text { Min-Max } \\
\text { Median (IQR) }\end{array}$ & $\begin{array}{c}3-15 \\
15(14-15)\end{array}$ & $\begin{array}{c}3-15 \\
11(8-13)\end{array}$ & $\mathrm{Z}_{(\mathrm{MW})=}=3.715$ & $0.000 *$ \\
\hline COHb level (\%) & $\begin{array}{c}\text { Min-Max } \\
\text { Median (IQR) }\end{array}$ & $\begin{array}{c}3-20 \\
10(6-12)\end{array}$ & $\begin{array}{c}2-48 \\
12(8-23)\end{array}$ & $\mathrm{Z}_{(\mathrm{MW})=-2.490}$ & $0.013^{*}$ \\
\hline QTc (msec) & $\begin{array}{c}\text { Min-Max } \\
\text { Median (IQR) }\end{array}$ & $\begin{array}{c}364-516 \\
413(403-465) \\
\end{array}$ & $\begin{array}{c}360-512 \\
430(409-467) \\
\end{array}$ & $\mathrm{Z}_{(\mathrm{MW})=}=-0.783$ & 0.433 \\
\hline $\begin{array}{c}\text { QTc interval (Category): } \\
\text { Normal } \\
\text { Prolonged (>440msec) }\end{array}$ & $\begin{array}{l}\text { N (\%) } \\
\text { N (\%) }\end{array}$ & $\begin{array}{l}18(62.1 \%) \\
11(37.9 \%)\end{array}$ & $\begin{array}{l}18(54.5 \%) \\
15(45.5 \%)\end{array}$ & $\begin{array}{l}\mathrm{X}_{(\mathrm{Y})(\mathrm{df}=1)}^{2}= \\
0.116\end{array}$ & 0.733 \\
\hline $\begin{array}{c}\text { ECG severity grading: } \\
\text { Normal } \\
\text { Minor } \\
\text { Moderate } \\
\text { Severe } \\
\end{array}$ & $\begin{array}{l}\text { N (\%) } \\
\text { N (\%) } \\
\text { N (\%) } \\
\text { N (\%) }\end{array}$ & $\begin{array}{c}13(44.8 \%) \\
2(6.9 \%) \\
14(48.3 \%) \\
0(0 \%) \\
\end{array}$ & $\begin{array}{c}8(24.2 \%) \\
4(12.1 \%) \\
19(57.6 \%) \\
2(6.1 \%)\end{array}$ & $\begin{array}{c}\mathrm{X}_{(\mathrm{MC})(\mathrm{df}=3)}= \\
4.375\end{array}$ & 0.248 \\
\hline $\begin{array}{c}\text { Length of hospital stay: } \\
<\text { 1day } \\
\text { 1-3days } \\
\text { > 3days } \\
\end{array}$ & $\begin{array}{l}N(\%) \\
N(\%) \\
N(\%)\end{array}$ & $\begin{array}{c}16(55.2 \%) \\
12(41.4 \%) \\
1(3.4 \%)\end{array}$ & $\begin{array}{c}8(24.2) \\
10(30.3 \%) \\
15(45.5 \%)\end{array}$ & $\begin{array}{c}\mathrm{X}_{(\mathrm{MC})(\mathrm{df}=2)}= \\
14.902\end{array}$ & $0.000 *$ \\
\hline $\begin{array}{c}\text { Number of needed HBO } \\
\text { sessions }\end{array}$ & $\begin{array}{c}\text { Min-Max } \\
\text { Median (IQR) }\end{array}$ & $\begin{array}{c}0-4 \\
1(0-1) \\
\end{array}$ & $\begin{array}{c}0-8 \\
4(1-5) \\
\end{array}$ & $\mathrm{Z}_{(\mathrm{MW})}=-3.498$ & $0.000 *$ \\
\hline
\end{tabular}

Min-Max: Minimum-Maximum; IQR: inter-quartile range ( $25^{\text {th }}-75^{\text {th }}$ percentile); \%: Percent within group;

*: Statistically significant ( $<<0.05)$; MC: Monte Carlo correction for Pearson's Chi Square $p$ value;

MW: Mann-Whitney test; Y: Yates correction for Pearson's Chi Square and its $p$ value; df: degree of freedom 
Table (4): Vital signs and arterial blood gases of DNS-complicated and non-complicated acute CO poisoned patients.

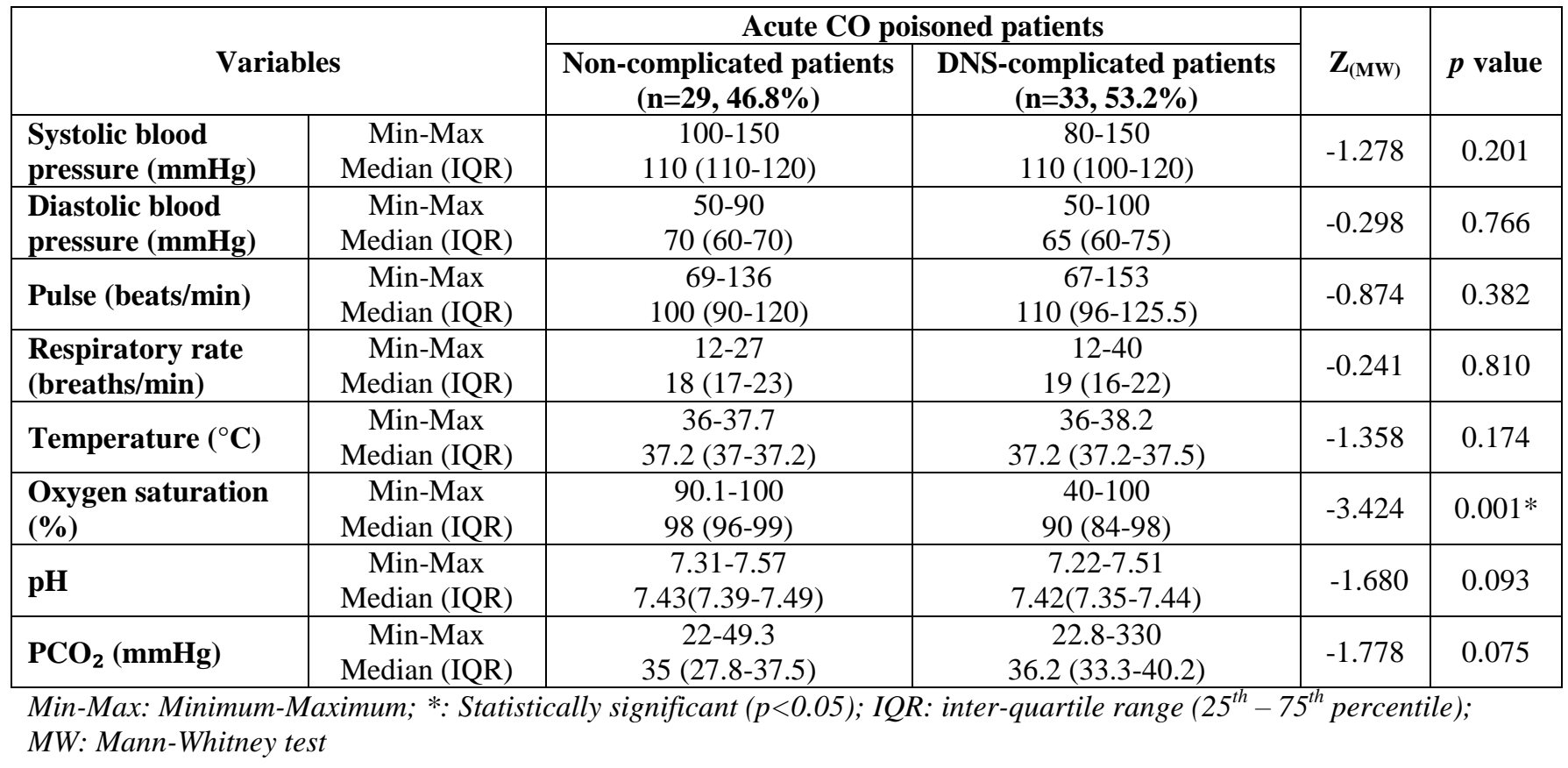

Table (5): Hematologic parameters based on peripheral CBC count of DNS-complicated and non-complicated acute CO poisoned patients.

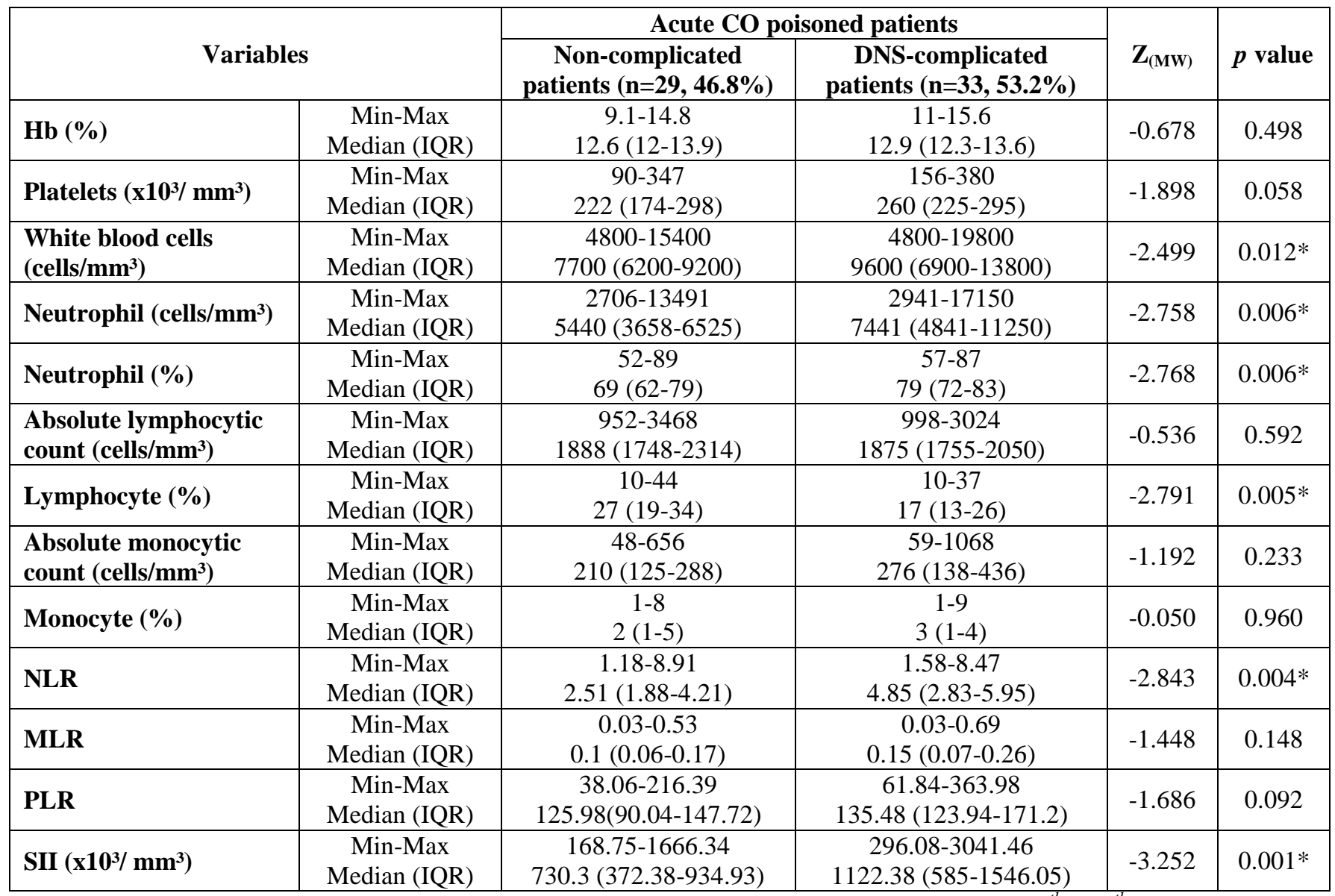

Min-Max: Minimum-Maximum; *: Statistically significant ( $p<0.05)$; IQR: inter-quartile range $\left(25^{\text {th }}-75^{\text {th }}\right.$ percentile) MW: Mann-Whitney test 


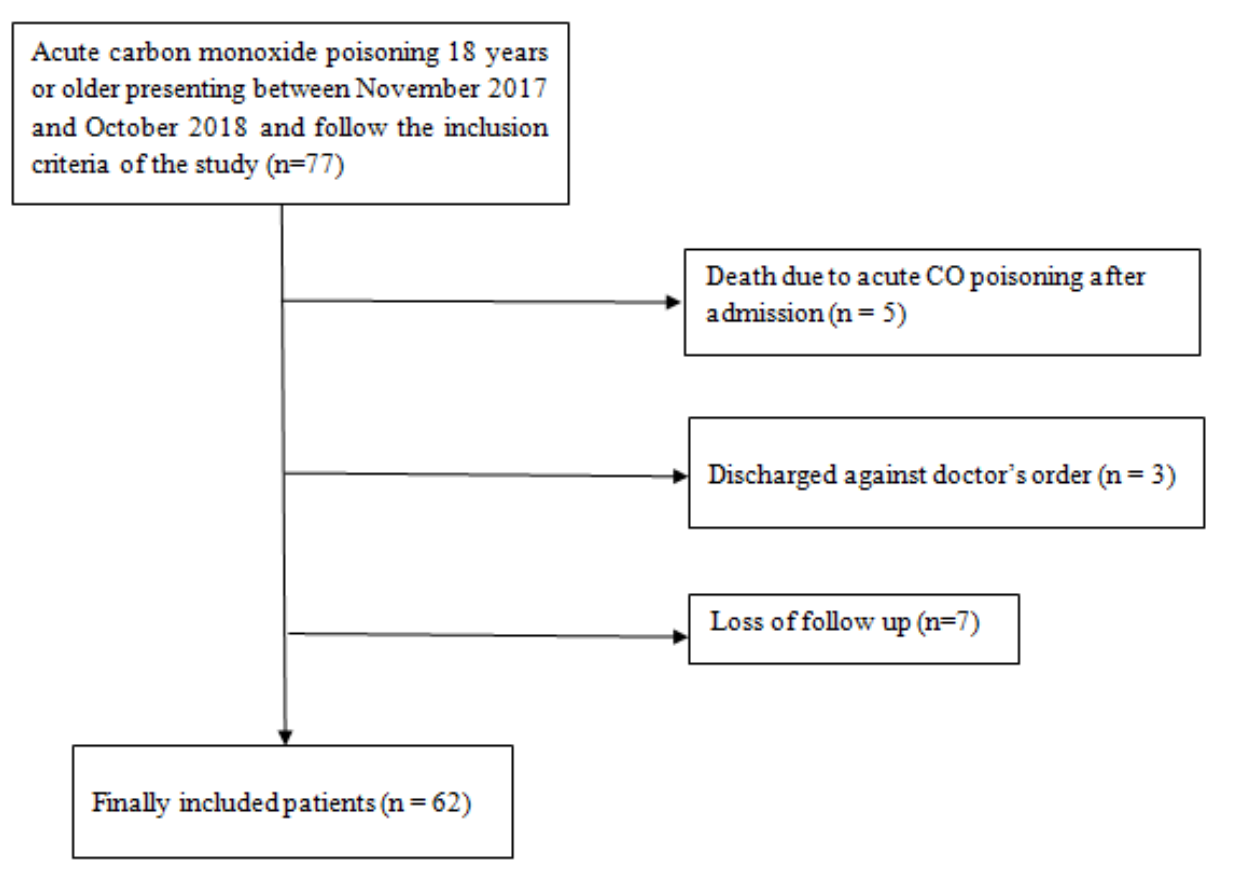

Figure (1): Algorithm for patient selection

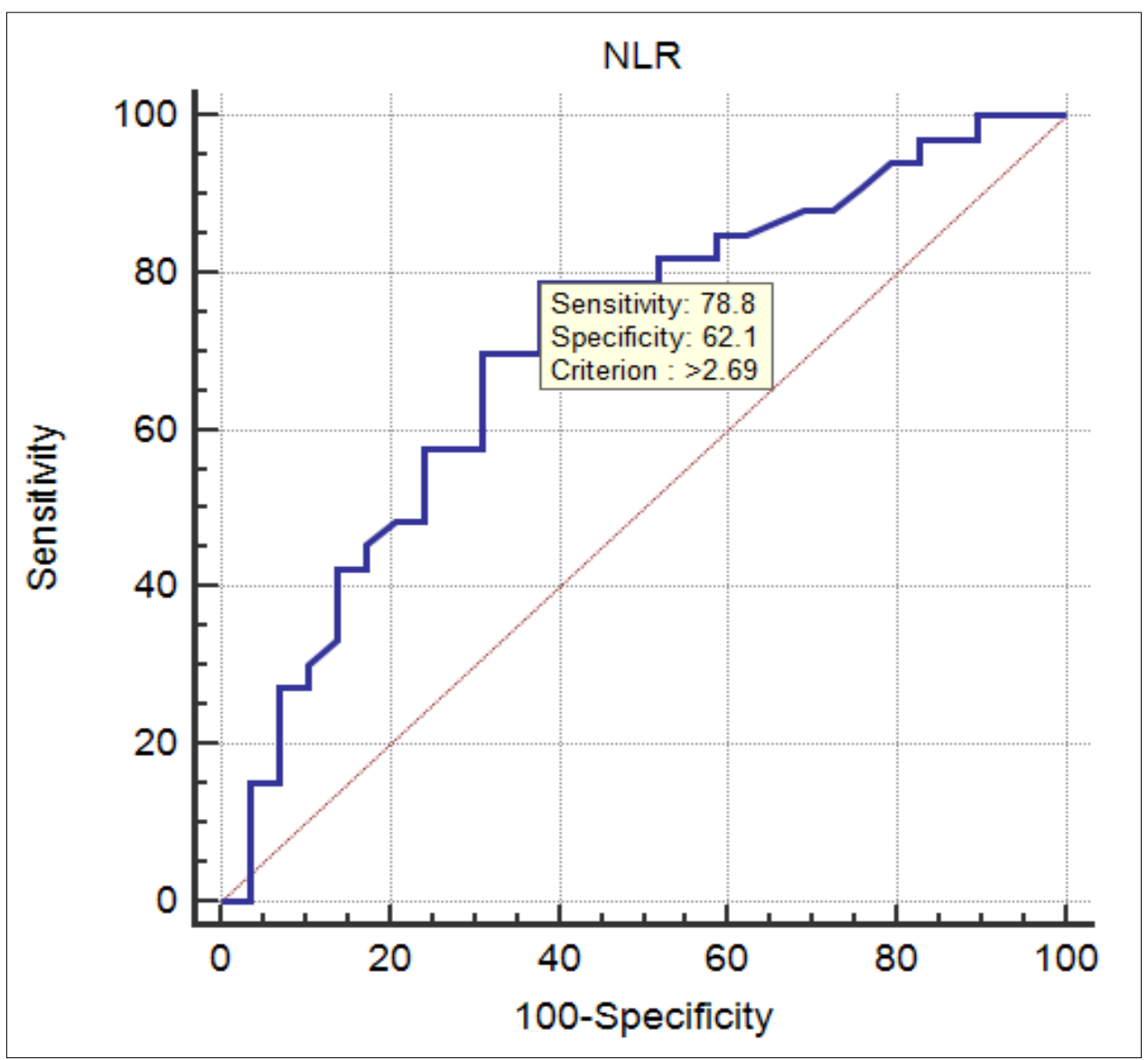

Figure (2): Area under ROC curve of NLR for discrimination of DNS in acute $\mathrm{CO}$ poisoned patients. 


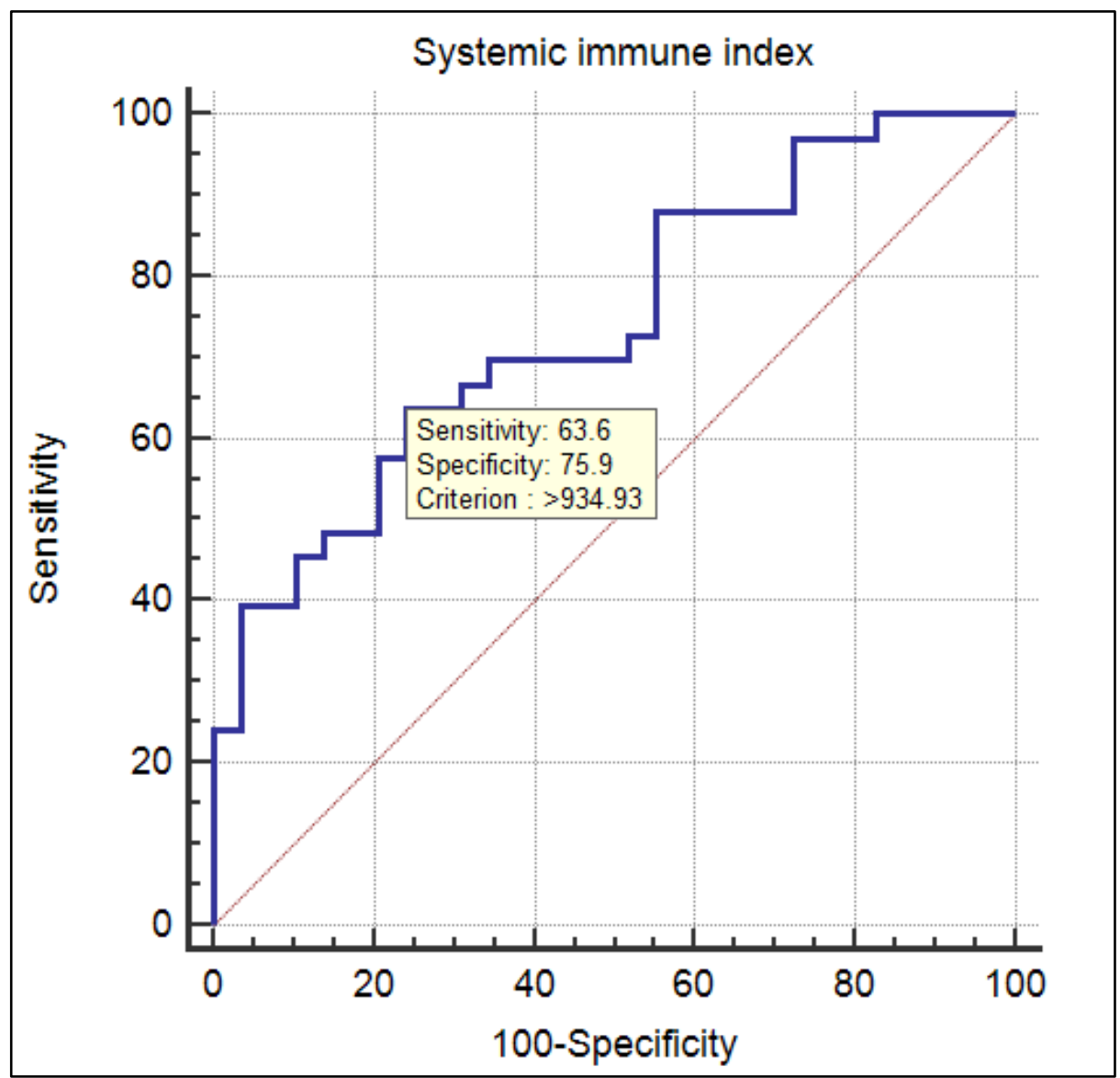

Figure (3): Area under ROC curve of SII for discrimination of DNS in acute $\mathrm{CO}$ poisoned patients.

\section{Discussion}

Acute CO poisoning is found as frequent emergency medical condition worldwide that have high morbidity and mortality (Sönmez et al., 2018). After CO intoxication, recurrent transient DNS may occur with alternating periods of exacerbation and remission. Moreover, DNS causes diffuse white matter or gray matter injury and may be reversible (Beppu et al., 2011; Kuroda et al., 2015). So, early prediction of DNS is very important in toxicological emergencies.

Nowadays, new hematological ratios based on peripheral CBC count are available as new inflammatory prognostic marker. Based on this, this study aimed to investigate whether hematological parameters based on peripheral CBC counts are associated with occurrence of DNS after acute CO poisoning and which parameters could predict them.

Concerning vital signs, this study showed that median of blood pressure (both systolic and diastolic) and oxygen saturation were significantly lower while QTc interval was significantly longer when comparing the included acute CO poisoned patients with control group. These were more or less in agreement with many previous observations in majority of centers in Egypt and across the world (Ismail et al., 2013; Abass et al., 2017; Liao et al., 2019b). The possible mechanism of QTc interval prolongation in CO poisoning is disruption of repolarization caused by late inward $\mathrm{Na}^{+}$current resulting in action potential prolongation (Dallas et al., 2012).

Regarding hematological parameters, total WBC count, absolute neutrophil count and percentage were significantly higher in acute $\mathrm{CO}$ poisoned patients compared to control group. This was in accordance with Karabacak et al. (2015) who found higher neutrophil count at presentation in severe $\mathrm{CO}$ poisoned patients compared to control group. This could be attributed to degranulation of intravascular neutrophils after acute CO poisoning, which induces generation of reactive oxygen species and catalyzes lipid peroxidation in human (Thom et al., 2006b). Moreover, neutrophils activation promotes the synthesis of inflammatory cytokines so; they are potential biomarkers for inflammation (Huang et al., 2018b).

On the other hand, lymphocytes percentage, absolute monocytic count and percentage were significantly lower in acute CO poisoned patients compared to control group in this study. This could be attributed to physiological stress leading to corticosteroids and catecholamine release after the activation of neurohormonal system to increase pro-inflammatory cytokine levels. These corticosteroids and catecholamine release in turn 
mediates lymphocyte apoptosis and lymphocytopenia in peripheral blood (Moon et al., 2019b).

Moreover, this study found that median values of NLR and SII were significantly higher in acute CO poisoned patients when compared to control group. Previous researches have detected NLR and SII as simple powerful markers of ongoing nonspecific inflammation by combining of independent parameters (neutrophils, lymphocytes, platelets) (Hu et al., 2014; Karabacak et al., 2015). This confirmed ongoing inflammation induced by $\mathrm{CO}$ poisoning that may play important role in the pathogenesis of CO complications included DNS.

The incidence of DNS in the studied acute CO patients was $53.2 \%$ which was more or less comparable with previous reports which indicted variable incidence of DNS in acute CO poisoned patients (Ku et al., 2010; Kudo et al., 2014; Du et al., 2019). These variations in the incidence of DNS could be attributed to various factors e.g. the studied patients' age, duration of $\mathrm{CO}$ exposure, delay time before hospitalization and $\mathrm{CO}$ concentration.

This neurological sequalae could be attributed to mitochondrial oxidative stress in the neuronal cells by free radicals generation, white matter demyelination by CO immune response, post ischemic reperfusion injury, apoptosis and inflammation (Khot et al., 2007; Sönmez et al., 2018). Moreover, abnormal inflammatory reaction of acute CO poisoning may be long lasting after the initial stage of poisoning (Lippi et al., 2012; Rose et al., 2017).

Regarding demographics, admission data, vital signs, QTc, ECG severity grading, COHb level, hospital stay and number of needed HBO sessions, DNS-complicated patients had significantly lower initial GCS and oxygen saturation and higher $\mathrm{COHb}$ level on admission, length of hospital stay and number of needed HBO sessions than non-complicated ones.

Initial low GCS at admission was reported as early predictor of DNS in acute CO poisoning in many previous studies (Ku et al., 2010; Kudo et al., 2014; Du et al., 2019). This may be due to CO induced neuronal injury by its hypoxic and inflammatory effect. This was in accordance with Cha et al. (2018) who concluded that patients who have disturbed consciousness in the acute phase of CO poisoning, can be easily complicated with DNS in the subacute or chronic phase.

Regarding COHB levels at admission, Cervellin et al. (2014) and Hampson (2018) demonstrated that elevated $\mathrm{COHb}$ levels are only exposure indicator and does not correlate with acute CO poisoning severity or neurological deficits. Furthermore, length of hospital stay was longer in the DNS-complicated patients; this was in accordance with Kudo et al. (2014) because these patients usually needed longer time to recover from the acute phase of $\mathrm{CO}$ poisoning.

Regarding treatment with $\mathrm{HBO}$ sessions, this study showed that DNS-complicated patients received more HBO sessions than non-complicated one. This means that the risk for DNS in patients who received HBO sessions was higher than that in patients who did not. This was in line with results of many previous studied assumed that the supplemental benefit of HBO therapy for reducing DNS has been a matter of debates (Annane et al., 2011; Hampson et al., 2012; Huang et al., 2017).

This could be explained by Huang et al. (2018a), who stated that HBO sessions had more prominent lifesaving effect than their possible DNS-reducing effect. This may be attributed to that $\mathrm{HBO}$ sessions were the main line of treatment in acute $\mathrm{CO}$ intoxicated patients who were also at higher risks for DNS but these patients have passed away before completed the needed number of sessions due to different treatment protocols (Hosseininejad et al., 2018). This confirms the fact that there must be easy, cheap and widely available laboratory marker of ongoing systemic inflammation in acute CO intoxicated patients to decide whether patient is in need for further HBO sessions or not before leaving hospital.

No statistical difference was detected between DNScomplicated and non-complicated patients regarding QTc interval and ECG severity grading in this study. On the other hand, Liao et al. (2018) found QTc interval significantly prolonged in DNS-complicated patients. These confounded results are reasonable because CO is eliminated through pulmonary circulation within about 5 hours under room air breathing (Chang et al., 2017). In our study, the delay time was ranged from 0.5 to 36 hours which could lead to pulmonary decontamination of CO.

This study had investigated different hematological parameters based on peripheral CBC counts (NLR; MLR; PLR and SII) as a predictor of DNS in acute CO poisoned patients and which parameters might significantly improve the predictive accuracy for DNS. Median values of NLR and SII were significantly higher in DNS-complicated patient than non-complicated ones in this study. Several studies demonstrated the relevance between ongoing inflammation and DNS after CO poisoning (Thom et al., 2004; Thom et al., 2006a;Thom et al., 2006b).

During the inflammatory process, leucocytes and platelets circulating in the blood act in conjunction with endothelial cells. Each subtype of leucocyte has a different role in the inflammatory and immunological process and contributes differently to the pathophysiology (Moon et al., 2019a). Platelets also contribute by releasing numerous inflammatory mediators that modify leucocyte and endothelial responses to a range of inflammatory stimuli (Thomas and Storey, 2015).

Based on ROC analysis, both NLR and SII were significant predictors of DNS in CO poisoned patients (AUCs: 0.711 and 0.741 respectively). This was in accordance with Moon et al. (2019a) who detected that AUCs of NLR and SII of CO poisoned patients over the first 12 hours after presentation were $>0.70$. The acceptable performance of these comprehensive inflammatory ratios may be attributed to that these ratios are indicating both the pro-inflammatory and immunosuppression status by integrating two or three 
types of circulating immune-inflammatory cells, such as neutrophils, monocytes, lymphocytes and platelets (Moon et al., 2019b). Neutrophils are markers of ongoing nonspecific inflammation while lymphocytes are markers of the regulatory pathway (Karabacak et al., 2015).

Moreover, these simple and cheap ratios can be measured easily at presentation by routine CBC count testing. They can be used by emergency physicians as early predictors of DNS in acute CO poisoned patients that are considered the most serious complication after apparent recovery from acute CO poisoning. This will help emergency physicians to decide more sufficient HBO sessions for those patients to decrease neurological insult and to prevent early discharge of patients from hospital before enough treatment.

\section{Conclusion}

This study demonstrated that NLR and SII ratios based on peripheral CBC count may be helpful predictors of DNS after acute CO poisoning. Both can be used as simple measureable promising parameters predicting DNS after acute CO poisoning.

\section{References}

Abass, M.A., Arafa, M.H., EL-shal, A.S., et al. (2017): Asymmetric dimethylarginine and heart-type fatty acid-binding protein 3 are risk markers of cardiotoxicity in carbon monoxide poisoning cases in Zagazig university hospitals. Hum. Exp. Toxicol. 36(3), 247-255.

Akdur, O., Durukan, P., Ozkan, S., et al. (2010): Poisoning severity score, Glasgow coma scale, corrected QT interval in acute organophosphate poisoning. Hum. Exp. Toxicol. 29(5), 419-425.

Annane, D., Chadda, K., Gajdos, P., et al. (2011): Hyperbaric oxygen therapy for acute domestic carbon monoxide poisoning: two randomized controlled trials. Intensive care med. 37(3), 486-492.

Beppu, T., Nishimoto, H., Fujiwara, S., et al. (2011): 1Hmagnetic resonance spectroscopy indicates damage to cerebral white matter in the subacute phase after CO poisoning. J. Neurol. Neurosurg. Psychiatry. 82(8), 869-875.

Cervellin, G., Comelli, I., Rastelli, G., et al. (2014): Initial blood lactate correlates with carboxyhemoglobin and clinical severity in carbon monoxide poisoned patients. Clin. Biochem. 47(18), 298-301.

Cevik, Y., Tanriverdi, F., Delice, O., et al. (2010): Reversible Increases in Qt Dispersion and P Wave Dispersion during Carbon Monoxide Intoxication. Hong Kong J. Emerg. Me. 17(5), 441-450.

Cha, Y., Kim, H., Do, H., et al. (2018): Serum neuronspecific enolase as an early predictor of delayed neuropsychiatric sequelae in patients with acute carbon monoxide poisoning. Hum. Exp. Toxicol. 37(3), 240-246.
Chang, Y.C., Lee, H.Y., Huang, J.L., et al. (2017): Risk Factors and Outcome Analysis in Children with Carbon Monoxide Poisoning. Pediatr. Neonatol. 58(2), 171-177.

Dallas, M.L., Yang, Z., Boyle, J.P., et al. (2012): Carbon monoxide induces cardiac arrhythmia via induction of the late $\mathrm{Na}+$ current. Am. J. Respir. Crit. Care Med. 186(7), 648-656.

Du, X., Gu, H., Hao, F., et al. (2019): Utility of brain CT for predicting delayed encephalopathy after acute carbon monoxide poisoning. Exp. Ther. Med. 17(4), 2682-2688.

Eichhorn, L., Thudium, M. \& Jüttner, B. (2018): The Diagnosis and Treatment of Carbon Monoxide Poisoning. Dtsch. Ärztebl. Int. 115(51-52), 863870.

Geng, Y., Shao, Y., Zhu, D., et al. (2016): Systemic Immune-Inflammation Index Predicts Prognosis of Patients with Esophageal Squamous Cell Carcinoma: A Propensity Score-matched Analysis. Sci. Rep. 6, 39482.

Guan, L., Li, Z.Y., Zhang, Y.L., et al. (2015): Early Biomarkers in $1 \mathrm{H}$ Nuclear Magnetic Resonance Spectroscopy of Striatal Pathological Mechanisms after Acute Carbon Monoxide Poisoning in Rats. Biomed. Environ. Sci. 28(10), 728-737.

Hafez, A.S. \& El-Sarnagawy, G.N. (2019): S-100beta in predicting the need of hyperbaric oxygen in COinduced delayed neurological sequels. Hum. Exp. Toxicol. e960327119897104.

Hampson, N.B. (2018): Carboxyhemoglobin: a primer for clinicians. Undersea. Hyperb. Med. 45(2), 165-171.

Hampson, N.B., Piantadosi, C.A., Thom, S.R., et al. (2012): Practice recommendations in the diagnosis, management, and prevention of carbon monoxide poisoning. Am. J. Respir. Crit. Care Med. 186(11), 1095-1101.

Hosseininejad, S.M., Aminiahidashti, H., Goli Khatir, I., et al. (2018): Carbon monoxide poisoning in Iran during 1999-2016: A systematic review and meta-analysis. J. Forensic Leg. Med. 53, 8796.

Hu, B., Yang, X.R., Xu, Y., et al. (2014): Systemic immune-inflammation index predicts prognosis of patients after curative resection for hepatocellular carcinoma. Clin. Cancer Res. 20(23), 6212-6222.

Huang, C.C., Ho, C.H., Chen, Y.C., et al. (2017): Hyperbaric Oxygen Therapy Is Associated With Lower Short- and Long-Term Mortality in Patients With Carbon Monoxide Poisoning. Chest. 152(5), 943-953. 
Huang, C.C., Ho, C.H., Chen, Y.C., et al. (2018a): Impact of Hyperbaric Oxygen Therapy on Subsequent Neurological Sequelae Following Carbon Monoxide Poisoning. J. Clin. Med. 7(10), 349.

Huang, Y., Ying, Z., Quan, W., et al. (2018b): The clinical significance of neutrophil-to-lymphocyte ratio and monocyte-to-lymphocyte ratio in Guillain-Barre syndrome. Int. J. Neurosci. 128(8), 729-735.

Huysal, K., Ustundag Budak, Y., Aydin, U., et al. (2016): $\mathrm{COHb}$ Level and High-Sensitivity Cardiac Troponin $\mathrm{T}$ in 2012 in Bursa, Turkey: A Retrospective Single-Center Study. Iran. Red Crescent Med. J. 18(5), e27061.

Ismail, M., El-Ghamry, H., Shaker, O., et al. (2013): Some biomarkers in carbon monoxide-induced cardiotoxicity. J. Environ. Anal. Toxicol. 3(4), 176.

Jeon, S.B., Sohn, C.H., Seo, D.W., et al. (2018): Acute Brain Lesions on Magnetic Resonance Imaging and Delayed Neurological Sequelae in Carbon Monoxide Poisoning. JAMA neurology. 75(4), 436-443.

Karabacak, M., Turkdogan, K.A., Coskun, A., et al. (2015): Detection of neutrophil-lymphocyte ratio as a serum marker associated with inflammations by acute carbon monoxide poisoning. J. Acute Dis. 4(4), 305-308.

Khot, S., Walker, M., Lacy, J.M., et al. (2007): An Unsuccessful Trial of Immunomodulatory Therapy in Delayed Posthypoxic Demyelination. Neurocrit. Care 7(3), 253-256.

Kim, J.H., Park, J.M., Kim, M.J., et al. (2015): Hematological Parameters Predicting Complications of Caustic Ingestion: A Retrospective Study. J. Korean. Soc. Emerg. Med. 26(6), 591-597.

Kim, Y.J., Sohn, C.H., Seo, D.W., et al. (2019): Clinical Predictors of Acute Brain Injury in Carbon Monoxide Poisoning Patients With Altered Mental Status at Admission to Emergency Department. Acad. Emerg. Med. 26(1), 60-67.

Ku, H.L., Yang, K.C., Lee, Y.C., et al. (2010): Predictors of carbon monoxide poisoning-induced delayed neuropsychological sequelae. Gen. Hosp. Psychiatry. 32(3), 310-314.

Kudo, K., Otsuka, K., Yagi, J., et al. (2014): Predictors for delayed encephalopathy following acute carbon monoxide poisoning. BMC Emerg. Med. 14(1), 3.

Kuroda, H., Fujihara, K., Kushimoto, S., et al. (2015): Novel clinical grading of delayed neurologic sequelae after carbon monoxide poisoning and factors associated with outcome. Neuro Toxicology. 48, 35-43.
Liao, S.C., Mao, Y.C., Hung, Y.M., et al. (2018): Predictive Role of QTc Prolongation in Carbon Monoxide Poisoning-Related Delayed Neuropsychiatric Sequelae. Biomed. Res. Int. 2018, 2543018.

Liao, S.C., Mao, Y.C., Yang, K.J., et al. (2019a): Targeting optimal time for hyperbaric oxygen therapy following carbon monoxide poisoning for prevention of delayed neuropsychiatric sequelae: A retrospective study. J. Neurol. Sci. 396, 187-192.

Liao, W.C., Cheng, W.C., Wu, B.R., et al. (2019b): Outcome and prognostic factors of patients treated in the intensive care unit for carbon monoxide poisoning. J. Formos. Med. Assoc. 118(4), 821-827.

Lin, M.S., Lin, C.C., Yang, C.C., et al. (2018): Myocardial injury was associated with neurological sequelae of acute carbon monoxide poisoning in Taiwan. J. Chin. Med. Assoc. 81(8), 682-690.

Lippi, G., Rastelli, G., Meschi, T., et al. (2012): Pathophysiology, clinics, diagnosis and treatment of heart involvement in carbon monoxide poisoning. Clin. Biochem. 45(16), 1278-1285.

Moon, J.M., Chun, B.J. \& Cho, Y.S. (2019a): The predictive value of scores based on peripheral complete blood cell count for long-term neurological outcome in acute carbon monoxide intoxication. Basic Clin. Pharmacol.Toxicol. 124(4), 500-510.

Moon, J.M., Chun, B.J., Cho, Y.S., et al. (2019b): Diagnostic Value of Parameters Related to White Blood Cell Counts for Troponin I Elevation in CO Poisoning. Cardiovasc. Toxicol. 19(4), 334-343.

Pang, L., Bian, M., Zang, X.X., et al. (2013): Neuroprotective Effects of Erythropoietin in Patients with Carbon Monoxide Poisoning. J. Biochem. Mol. Toxicol. 27(5), 266-271.

Pepe, G., Castelli, M., Nazerian, P., et al. (2011): Delayed neuropsychological sequelae after carbon monoxide poisoning: predictive risk factors in the Emergency Department. A retrospective study. Scand. J. Trauma. Resusc. Emerg. Med. 19(1), 16.

Postema, P.G. \& Wilde, A.A.M. (2014): The measurement of the QT interval. Curr. Cardiol. Rev. 10(3), 287-294.

Rose, J.J., Wang, L., Xu, Q., et al. (2017): Carbon Monoxide Poisoning: Pathogenesis, Management, and Future Directions of Therapy. Am. J. Respir. Crit. Care. Med. 195(5), 596-606.

Sönmez, B.M., İşcanlı, M.D., Parlak, S., et al. (2018): Delayed neurologic sequelae of carbon monoxide intoxication. Turk. J. Emerg. Med. 18(4), 167-169. 
Thom, S.R., Bhopale, V.M., Fisher, D., et al. (2004): Delayed neuropathology after carbon monoxide poisoning is immune-mediated. Proc. Natl. Acad. Sci. U.S.A. 101(37), 13660-13665.

Thom, S.R., Bhopale, V.M. \& Fisher, D. (2006a): Hyperbaric oxygen reduces delayed immunemediated neuropathology in experimental carbon monoxide toxicity. Toxicol. Appl. Pharmacol. 213(2), 152-159.

Thom, S.R., Bhopale, V.M., Han, S.T., et al. (2006b): Intravascular neutrophil activation due to carbon monoxide poisoning. Am. J. Respir. Crit. Care. Med. 174(11), 1239-1248.
Thomas, M.R. \& Storey, R.F. (2015): The role of platelets in inflammation. Thromb. Haemost. 114(3), 449-458.

Vezzani, G., Socias, S., Bianco, A., et al. (2016): Inflammatory mediators and other biomarkers in co-intoxicated patients after hyperbaric oxygen therapy (HBO2). Acta. Med. 32, 189.

Wang, I.J., Yeom, S.R., Park, S.W., et al. (2019): Poison severity score and sequential organ failure assessment score: Carbon monoxide poisoning prognosis. PloS one. 14(3), e0212025.

\title{
العوامل الدموية كمتنبؤات مبكرة للعواقب العصبية المتأخرة في التسمم الحاد بأول أكسيد الكربون
}

\author{
مروة عمد شاهين' و الزهراء عبد العظيم علام' و رشا عادل الحولى و هبه ابراهيم لاشين' \\ الملخص العربى
}

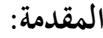

المقدمة: تعد العواقب العصبية المتأخرة (DNS) المرض الأكثر شيوعًا بعد التسمم الحاد بأول أكسيد الكربون. لذلك ، فإن الوقاية والتنبؤ المبكر هما الهدف

الرئيسي للعلاج.الهدف من الدراسة: محف هذه الدراسة إلى فحص عدد من العوامل على أساس عدد خلايا الدم الكاملة (CBC) كمتنبئات للعواقب

العصبية المتأخرة بعد التسمم الحاد بأول أكسيد الكربون. طريقة البحث: شملت الدراسة VT من مرضى التسمم الحاد بأول أكسيد الكربون و رب متطوعًا صحيًا.

عند دخول المستشفى، تم أخذ التاريخ المرضي والفحص السريري والفحوصات المخحبرية الروتينية. تم قياس النسب على أساس عدد خلايا الدم الكاملة، تمت متابعة

المرضى لمدة ج أشهر لإشارات العواقب العصبية المتأخرة. النتائج: قد أظهرت النتائج أن إجمالي عدد خلايا الدم البيضاء (WBC) العد المطلق لخلايا النيوترفيل

والنسبة المئوية والقيم المتوسطة لنسبة خلايا النيوتروفيل للخلايا اللمفاوية (NLR) ومؤشر الالتهاب المناعي العام ( SII) أعلى بشكل ملحوظ بينما كانت نسبة

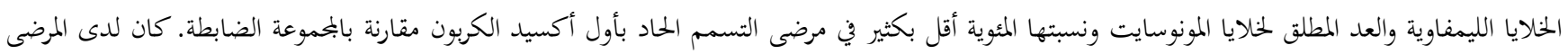

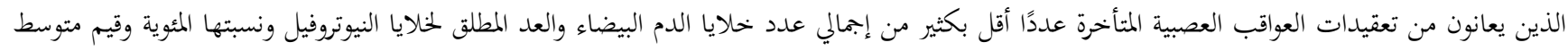

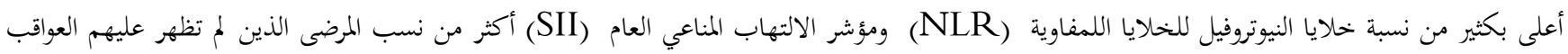

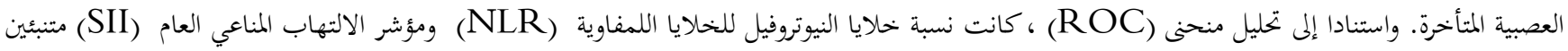

مهمين للعواقب العصبية المتأخرة (DNS).الخلاصة: تم استنتاج أن نسبة خلايا النيوتروفيل للخلايا اللمفاوية (NLR) و مؤشر الالتهاب المناعي العام (SII) ) قد تكون عوامل مساعدة في التنبؤ بالعواقب العصبية المتأخرة (DNS) بعد التسمم الحاد بأول أكسيد الكربون. 
